OPEN ACCESS

Edited by: Luis Sobrevia,

Pontificia Universidad Católica de Chile, Chile

Reviewed by:

Enrique Guzmán-Gutiérrez, University of Concepcion, Chile Fabian N. Pardo,

Universidad de Valparaiso, Chile

*Correspondence:

Yufan Wang

yyffwang@sina.com

${ }^{t}$ These authors have contributed equally to this work and share first authorship

Specialty section: This article was submitted to

Clinical Diabetes, a section of the journal

Frontiers in Endocrinology

Received: 28 October 2021 Accepted: 02 February 2022 Published: 07 March 2022

Citation:

Gong Y, Li N, Lai M, Fang F, Yang J, Kang $M$, Shen $T$, Peng $Y$ and Wang $Y$

(2022) Consistently Low Levels of Osteocalcin From Late Pregnancy to Postpartum Are Related to Postpartum Abnormal Glucose Metabolism in GDM Patients.

Front. Endocrinol. 13:803624. doi: 10.3389/fendo.2022.803624

\section{Consistently Low Levels of Osteocalcin From Late Pregnancy to Postpartum Are Related to Postpartum Abnormal Glucose Metabolism in GDM Patients}

\author{
Yujia Gong ${ }^{1 \dagger}$, Na Li ${ }^{1 \dagger}$, Mengyu Lai ${ }^{1}$, Fang Fang ${ }^{1}$, Jiaying Yang ${ }^{1}$, Mei Kang ${ }^{2}$, \\ Tingting Shen ${ }^{1}$, Yongde Peng ${ }^{1}$ and Yufan Wang ${ }^{1 *}$ \\ ${ }^{1}$ Department of Endocrinology and Metabolism, Shanghai General Hospital, Shanghai Jiao Tong University School of \\ Medicine, Shanghai, China, ${ }^{2}$ Clinical Research Center, Shanghai General Hospital, Shanghai Jiao Tong University School of \\ Medicine, Shanghai, China
}

Objective: Increasing evidence suggests that osteocalcin (OC), a marker of bone formation, plays an important role in glucose homoeostasis. Few studies have investigated the relationship between $\mathrm{OC}$ levels in gestational diabetes mellitus (GDM) patients and their postpartum glucose metabolism. This study evaluated the relationship between $\mathrm{OC}$ levels in late pregnancy, their longitudinal changes, and postpartum glucose metabolism among GDM patients.

Measures: Serum $\mathrm{OC}$ was measured in late pregnancy and the postpartum period for 721 GDM patients. All patients underwent a $75-\mathrm{g}$ oral glucose tolerance test (OGTT) at 68 weeks postpartum. According to postpartum OGTT outcomes, patients were categorized into abnormal glucose metabolism (AGM) ( $n=255)$ and normal glucose tolerance (NGT) groups ( $n=466$ ). Glucose metabolism-related indices were measured and calculated. Logistic regression analysis and linear mixed-effects model were used to assess the association between $\mathrm{OC}$ and postpartum AGM.

Results: In late pregnancy, OC levels were lower in the AGM group than in the NGT group $(13.93 \pm 6.90$ vs $15.33 \pm 7.63 \mathrm{ng} / \mathrm{ml}, P=0.015)$. After delivery, OC levels increased in both groups. However, OC levels remained lower in the AGM group than in the NGT group $(23.48 \pm 7.84$ vs $25.65 \pm 8.37 \mathrm{ng} / \mathrm{ml}, P=0.001)$. Higher OC levels in late pregnancy were associated with decreased risk of progressing to postpartum AGM (OR:0.96, 95\% Cl:0.94-0.99). Linear mixed-effects analysis showed that postpartum AGM patients exhibited consistently lower OC levels than NGT group from late pregnancy to the postpartum period after adjustment for cofactors ( $\beta=-1.70,95 \% \mathrm{Cl}$ : $-2.78--0.62)$. 
Conclusions: In GDM patients, consistently low levels of $O C$ from late pregnancy to postpartum were associated with increased postpartum AGM risk. The increase in serum OC may act as a protective factor to curb the progression of AGM at postpartum for GDM patients.

Keywords: osteocalcin, gestational diabetes mellitus, abnormal glucose metabolism, risk factors, postpartum glucose metabolism

\section{INTRODUCTION}

Recently, bone has been identified as an endocrine organ involved in energy metabolism through the secretion of specific hormones $(1,2)$. Osteocalcin (OC), a small noncollagenous protein of 49 amino acids that is exclusively secreted by osteoblasts, participates in bone remodeling and calcium homeostasis. OC has three $\gamma$-carboxyglutamic acid residues in the 17, 21 and 24 positions of its peptide chain, which undergoes a posttranslational modification at the glutamate residue to attain a higher affinity for hydroxyapatite to integrate into the bone extracellular matrix $(1,3,4)$. However, the undercarboxylated form (ucOC), as a bioactivator released into the circulation, may serve as a modulator of energy metabolism. Since ucOC levels are difficult to measure, most studies have focused on total OC (5-7).

Accumulating evidence shows that $\mathrm{OC}$ is vital in the crosstalk between bone remodeling and energy metabolism. Extensive animal studies have shown that OC stimulates insulin secretion directly by exerting an effect on pancreatic $\beta$-cell and indirectly via the secretion of glucagon-like peptide (GLP-1) by enteroendocrine $L$ cells leading to improved insulin sensitivity $(8,9)$. In contrast, osteocalcin-deficient mice displayed decreased $\beta$-cell proliferation, glucose intolerance, and insulin resistance (10). To date, almost human studies have supported the findings of animal studies. In humans, serum OC was reported to be decreased in patients with type 2 diabetes compared to the levels in nondiabetic controls; inversely associated with blood glucose levels, $\mathrm{HbAlc}, \mathrm{BMI}$ and insulin resistance; and positively associated with insulin secretion and insulin sensitivity $(7,11,12)$.

Gestational diabetes mellitus (GDM), defined as hyperglycemia first recognized during pregnancy, is one of the most common metabolic complications in pregnancy $(13,14)$. Although glucose intolerance in many GDM patients usually reverts to normal after delivery, these patients and their offspring face an increased lifetime risk of developing type 2 diabetes mellitus (T2DM) and cardiovascular diseases (CVD) in the future (15-17). As T2DM can be prevented or delayed by intensive lifestyle or metformin intervention $(18,19)$, it is suggested that GDM patients should be routinely screened, which is beneficial for early intervention (20).

A few studies have previously assessed the contribution of serum OC in this context. Higher OC concentrations in GDM patients than in euglycemic pregnant women and a positive association between OC and insulin resistance parameters during pregnancy had been reported (21-23), and these findings are in contrast to what has been observed in the context of diabetes. A possible explanation for the opposite results in GDM could be an early adaption to impaired glucose tolerance.

Although a few studies have explored the effects of OC on glucose metabolism in GDM patients, the role of OC levels in the postpartum glucose metabolism of GDM is unclear. Therefore, we studied 721 GDM patients and evaluated OC levels both in late pregnancy and postpartum. The associations of OC levels and their longitudinal trajectory changes with postpartum glucose metabolism of GDM were explored in our study.

\section{MATERIALS AND METHODS}

\section{Patient Population}

This retrospective study was performed at the Department of Endocrinology and Metabolism of Shanghai General Hospital from December 2015 to December 2020. Pregnant women underwent a 75-g OGTT test at 24-28 weeks of gestation and the International Association of Diabetes and Pregnancy Study Groups (IADPSG) criteria was used for the diagnosis of GDM (24): fasting plasma glucose (FPG) value $\geq 5.1 \mathrm{mmol} / \mathrm{L}$ and/or 1 $\mathrm{h}$ postprandial glucose ( $1 \mathrm{~h}-\mathrm{PG}$ ) value $\geq 10.0 \mathrm{mmol} / \mathrm{L}$ and/or $2-\mathrm{h}$ postprandial glucose ( $2 \mathrm{~h}-\mathrm{PG}$ ) value $\geq 8.5 \mathrm{mmol} / \mathrm{L}$. After delivery, all individuals with GDM were invited to undergo a 75-g OGTT test again at 6-8 weeks postpartum. Subjects with a history of diabetes mellitus (DM) or impaired fasting glucose (IFG) and/or impaired glucose tolerance (IGT) before pregnancy or lack of OC either in late gestation or at postpartum were excluded. Finally, a total of 721 subjects were included in this study.

Subjects were categorized into two groups according to 75 -g OGTT results at 6-8 weeks postpartum based on 1999 WHO criteria (25): 1. Abnormal glucose metabolism (AGM) group: IFG $(6.1 \mathrm{mmol} / \mathrm{L} \leq \mathrm{FPG}<7.0 \mathrm{mmol} / \mathrm{L}$ and $2 \mathrm{~h}-\mathrm{PG}<7.8 \mathrm{mmol} / \mathrm{L})$ or IGT $($ FPG $<7.0 \mathrm{mmol} / \mathrm{L}$ and $7.8 \mathrm{mmol} / \mathrm{L} \leq 2 \mathrm{~h}-\mathrm{PG}<11.1$ $\mathrm{mmol} / \mathrm{L}$ ) or DM (FPG $\geq 7.0 \mathrm{mmol} / \mathrm{L}$ or/and $2 \mathrm{~h}-\mathrm{PG} \geq 11.1 \mathrm{mmol} /$ L); 2. Normal glucose tolerance (NGT) group: FPG $<6.1 \mathrm{mmol} / \mathrm{L}$ and $2 \mathrm{~h}-\mathrm{PG}<7.8 \mathrm{mmol} / \mathrm{L}$.

This study was approved by the institutional ethics committee of Shanghai General Hospital.

\section{Study Protocol and Methods}

Clinical data including age at present pregnancy, family history of diabetes, parity, pregestational body mass index (pre-BMI), BMI at 6-8 weeks postpartum, OC levels and other clinical indexes of glucose and lipid metabolism were recorded. BMI was calculated as the weight in kilograms divided by the square of the 
height in meters $\left(\mathrm{kg} / \mathrm{m}^{2}\right)$. Homeostasis model assessment was used to estimate insulin resistance (HOMA-IR) which was defined as [fasting insulin $(\mu \mathrm{U} / \mathrm{ml}){ }^{*}$ fasting glucose $\left.(\mathrm{mmol} / \mathrm{l})\right] /$ 22.5 , and HOMA of $\beta$-cell (HOMA- $\beta$ ) index was used to assess $\beta$-cell function, which was calculated as $\left[20^{\star}\right.$ fasting insulin $(\mu \mathrm{U} /$ $\mathrm{ml})] /[$ fasting glucose $(\mathrm{mmol} / \mathrm{l})-3.5]$.

All blood samples were obtained in the morning after an overnight fast of $8-10 \mathrm{~h}$. In our study, we used $\mathrm{N}$-terminal midfragment of OC (N-MID OC), the largest proteolytic fragment with a relatively long half-life, to reflect serum OC levels (26). NMID OC was measured using electrochemiluminescent immunoanalysis (Roche Cobas e601, Germany). HbA1c was measured with an autoanalyzer (Lifotronic H8, Japan). Serum insulin was measured using an automated chemiluminescence systems (Abbott i2000, United States). Serum glucose and lipid profiles including serum total cholesterol (TC), triglycerides (TGs), high-density lipoprotein cholesterol (HDL-C) and lowdensity lipoprotein cholesterol (LDL-C), were measured with an automatic biochemistry analyzer (Siemens ADVIA2400, Germany). During the study period, instruments or testing methodologies unchanged.

\section{Statistical Analysis}

Data are presented as the mean \pm standard deviation (SD) or median (interquartile range, 25-75\%) for continuous variables and proportion for categorical variables, respectively. Normally distributed continuous variables were compared by Student's $t$ test, while nonnormally distributed continuous variables were analyzed by the Mann-Whitney $U$ test. Categorical variables were analyzed by $\chi^{2}$ test. The log-transformed levels of HOMA- $\beta$ were parameterized as a continuous variable. The Pearson correlation coefficients were calculated to assess the strength of the correlation of $\mathrm{OC}$ in late gestation and glucose related indicators, insulin resistance and $\beta$-cell function. Multivariate linear regression was performed to determine the associations between OC levels and insulin resistance, and $\beta$-cell function.

Multiple logistic regression models were used to calculate the odds ratio (OR) and 95\% CIs for the risk of postpartum AGM for OC levels in late gestation. A priori selection of conventional postpartum AGM risk factors, including age, postpartum BMI, parity, family history of diabetes and $\mathrm{HbAlc}$ in late pregnancy, was assessed at study enrollment. A linear mixed-effects model was performed to compare the longitudinal trajectories of OC in late gestation and postpartum in individuals with different postpartum glucose status according to 75-g OGTT results by using restricted maximum likelihood estimation. The model included serum OC in late pregnancy and postpartum, groups of different postpartum OGTT outcomes and time in late pregnancy and at postpartum. OC levels were adjusted for maternal age, prepregnancy BMI, family history of diabetes, parity and $\mathrm{HbAlc}$ in late gestation via covariate adjustment (fixed effects in the mixed model).

All statistical analyses were performed using SPSS version 26 (IBM Corp., Armonk, NY), and a $P$ value $<0.05$ was considered statistically significant.

\section{RESULTS}

The mean age in the cohort was $31.98 \pm 4.4$ years. All GDM patients received lifestyle modification and 174 (24\%) women received additionally insulin therapy during pregnancy. According to the results of the postpartum OGTT, 255 individuals were diagnosed with AGM, of whom 221 had IFG and/or IGT and 34 had diabetes. The remaining 466 women had normal glucose tolerance.

The baseline characteristics of the GDM subjects stratified by the outcomes of postpartum 75-g OGTT were shown in Table 1. Compared with the NGT group, subjects in the AGM group were older and had higher BMI and HbAlc both before and after delivery. Meanwhile, postpartum FBG, 2h-PG, 2h-INS, TC, TGs and LDL-C were significantly higher in the AGM group. Indices of insulin resistance (HOMA-IR) indicated that postpartum AGM women were more insulin resistant than NGT women. The OC levels of the AGM group were lower both in late pregnancy (NGT vs AGM: $15.33 \pm 7.63 \mathrm{ng} / \mathrm{ml}$ vs $13.93 \pm 6.90$ $\mathrm{ng} / \mathrm{ml}, P=0.015)$ and postpartum (NGT vs AGM: $25.65 \pm 8.37$ $\mathrm{ng} / \mathrm{ml}$ vs $23.48 \pm 7.84 \mathrm{ng} / \mathrm{ml}, P=0.001$ ) (Table $\mathbf{1}$ ).

The correlation analysis showed that OC levels were positively associated with postpartum FINS ( $\mathrm{r}=0.109, P=0.003$ ), HOMA-IR $(\mathrm{r}=0.098, P=0.008)$ and $\lg ($ HOMA- $\beta)(\mathrm{r}=0.132$, $P<0.001$ ) (Figure 1), but had no relationship with postpartum FBG and HbA1c (data not shown). In order to further explore the relationship between OC levels and insulin resistance and $\beta$ cell function, multivariate linear regression was used. We found that $\lg$ (HOMA- $\beta$ ) was positively associated with OC levels after adjusted for age, postpartum BMI, parity, family history of diabetes and HbA1c in late pregnancy $(\beta=0.003, P=0.015)$, while HOMA-IR was not associated with OC after adjusted covariates above $(\beta=0.002, P=0.783)$.

Logistic regression analysis revealed that the risk of developing AGM at postpartum was decreased by 3\% after adjusting for age and parity $(\mathrm{OR}=0.97,95 \% \mathrm{CI}$ : $0.95-0.99)$. This association remained significant after further adjustment for postpartum BMI, family history of diabetes and HbA1c in late pregnancy (OR=0.96, 95\%CI: 0.94-0.99) (Table 2).

OC levels increased significantly after delivery in both the NGT (from $15.33 \pm 7.63$ in late gestation to $25.65 \pm 8.37$ at postpartum, $P<0.001$ ) and AGM (from $13.93 \pm 6.90$ in late gestation to $23.49 \pm 7.84$ at postpartum, $P<0.001$ ) groups (Figure 2). Meanwhile, the linear mixed-effects model showed that the OC levels from late pregnancy to postpartum were consistently lower in AGM group than in NGT group, adjusted for parity, age, time points (late pregnancy and postpartum), prepregnancy BMI, HbA1c and family history of diabetes $(\beta=-1.70$, 95\% CI: -2.78- -0.62) (Supplementary Table 1).

\section{DISCUSSION}

In the current study, we investigated the association between serum OC levels and the postpartum glucose metabolism of GDM. We found that low serum OC in late pregnancy was 
TABLE 1 | Characteristics and metabolic parameters of GDM women with different glucose outcomes according to the 75-g OGTT at 6-8 weeks postpartum.

\begin{tabular}{|c|c|c|c|}
\hline & \multicolumn{2}{|c|}{$75-\mathrm{g}$ OGTT results at $6-8$ weeks postpartum } & \multirow[t]{2}{*}{$P$ value } \\
\hline & NGT $(n=466)$ & AGM $(n=255)$ & \\
\hline Age (years) & $31.62 \pm 4.17$ & $32.64 \pm 4.74$ & 0.004 \\
\hline Family history of diabetes & $101(21.7 \%)$ & 52 (20.6\%) & 0.715 \\
\hline Primiparity & 245 (53.0\%) & $128(50.6 \%)$ & 0.533 \\
\hline Pre-BMl $\left(\mathrm{kg} / \mathrm{m}^{2}\right)$ & $22.50 \pm 3.43$ & $23.25 \pm 3.56$ & 0.006 \\
\hline Postpartum BMI $\left(\mathrm{kg} / \mathrm{m}^{2}\right)$ & $23.72 \pm 3.21$ & $24.22 \pm 3.32$ & 0.046 \\
\hline HbA1c in late pregnancy (\%) & $5.45 \pm 0.44$ & $5.56 \pm 0.51$ & 0.005 \\
\hline OC level in late pregnancy (ng/ml) & $15.33 \pm 7.63$ & $13.93 \pm 6.90$ & 0.015 \\
\hline \multicolumn{4}{|l|}{ Laboratory values at postpartum } \\
\hline $\mathrm{FBG}(\mathrm{mmol} / \mathrm{L})$ & $4.91 \pm 0.52$ & $5.24 \pm 0.85$ & $<0.001$ \\
\hline 2h-PG (mmol/L) & $6.33 \pm 0.90$ & $9.20 \pm 1.52$ & $<0.001$ \\
\hline $\mathrm{TCH}(\mathrm{mmol} / \mathrm{L})$ & $5.30 \pm 0.96$ & $5.48 \pm 0.95$ & 0.017 \\
\hline TGs (mmol/L) & $1.19 \pm 0.78$ & $1.37 \pm 0.97$ & 0.01 \\
\hline HDL-C (mmol/L) & $1.49 \pm 0.38$ & $1.47 \pm 0.34$ & 0.535 \\
\hline LDL-C (mmol/L) & $3.01 \pm 0.81$ & $3.15 \pm 0.85$ & 0.027 \\
\hline FINS (mU/L) & $6.65 \pm 4.30$ & $7.33 \pm 4.90$ & 0.064 \\
\hline 2h-INS (mU/L) & $22.44(15.47,32.85)$ & $37.96(24.47,56.64)$ & $<0.001$ \\
\hline HOMA-IR & $1.47 \pm 1.00$ & $1.77 \pm 1.35$ & 0.002 \\
\hline HOMA- $\beta$ & $84.48(57.47,130.43)$ & $78.29(53.54,115.03)$ & 0.070 \\
\hline $\mathrm{HbA1c}(\%)$ & $5.36 \pm 0.43$ & $5.55 \pm 0.52$ & $<0.001$ \\
\hline OC at postpartum (ng/ml) & $25.65 \pm 8.37$ & $23.48 \pm 7.84$ & 0.001 \\
\hline
\end{tabular}

Data are presented as the mean $\pm S D$, median (interquartile range), or $n$ (\%) as appropriate.

OGTT, oral glucose tolerance test; NGT, normal glucose tolerance; AGM, abnormal glucose metabolism; Pre-BMI, body mass index before pregnancy; HbA1C, glycated haemoglobin; OC, osteocalcin; FBG, fasting blood glucose; $2 h-P G$, 2- h postprandial glucose; TCH, total cholesterol; TGs, triglycerides; HDL-C, high-density lipoprotein cholesterol; LDL-C, low-density

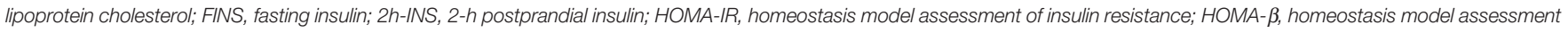
of beta-cell function.
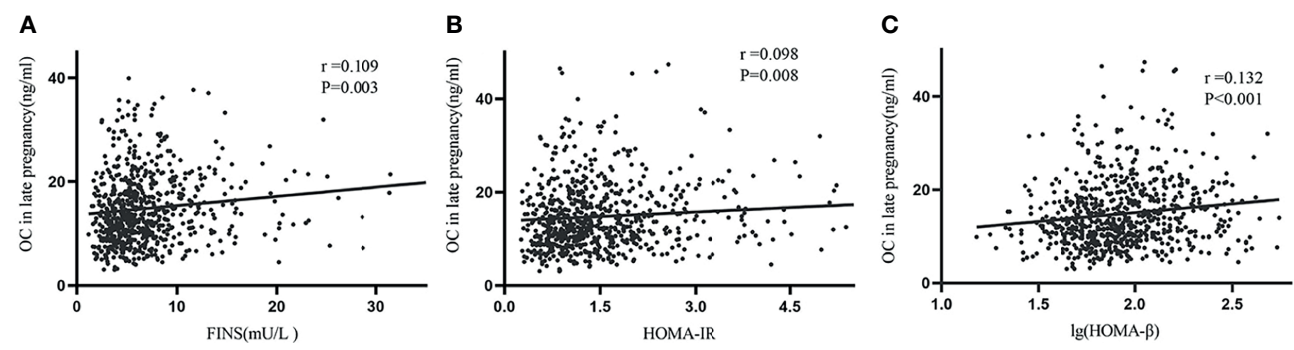

FIGURE 1 | Simple correlations between late pregnancy OC and postpartum FINS, HOMA-IR, and Ig (HOMA- $\beta$ ). Serum OC in late pregnancy was positively associated with FINS (A), HOMA-IR (B) and Ig (HOMA- $\beta$ ) (C).

TABLE 2 | Logistic regression analysis showing the association between OC in late pregnancy and postpartum AGM.

\begin{tabular}{lccc}
\hline Factors & OR & $\mathbf{9 5 \%} \mathbf{C l}$ & P value \\
\hline Age (years) & 1.05 & $1.01-1.1$ & 0.018 \\
Postpartum BMl $\left(\mathrm{kg} / \mathrm{m}^{2}\right)$ & 1.04 & $0.99-1.1$ & 0.126 \\
Family history of diabetes: no (reference) & 0.89 & $0.6-1.33$ & 0.573 \\
Parity: 1 (reference) & 0.80 & $0.55-1.16$ & 0.243 \\
OC level in late pregnancy $(\mathrm{ng} / \mathrm{ml})$ & 0.96 & $0.94-0.99$ & 0.004 \\
HDA1c in late pregnancy $\%)$ & 1.61 & $1.11-2.34$ & 0.012 \\
\hline
\end{tabular}

$B M I$, body mass index; OC, osteocalcin; HbA1c, glycated haemoglobin.

associated with increased risk of developing postpartum AGM. After delivery OC levels increased significantly in both groups, however, the OC levels were consistently low from late pregnancy to postpartum in the AGM group than in the NGT group. To the best of our knowledge, this is the first study exploring the relationship between longitudinal changes in OC and the postpartum glucose metabolism of GDM.

In animal and clinical investigations, OC, a traditional bone formation marker, has been found to participate in the regulation of glucose metabolism. Some evidence from animal studies suggested that higher OC concentrations are protective against diet-induced obesity and type 2 diabetes. Mice lacking the Esp gene, which encoded osteotesticular protein tyrosine phosphatase (OST-PTP), a receptor-like protein that inhibited the bioactivity of osteocalcin, exhibited hypoglycemia and were protected from glucose intolerance due to increases in pancreatic $\beta$-cell proliferation, insulin secretion and insulin sensitivity (10). In contrast, Osteocalcin- knockout mice had the opposite phenotypes, namely glucose intolerance and obesity (10). On 

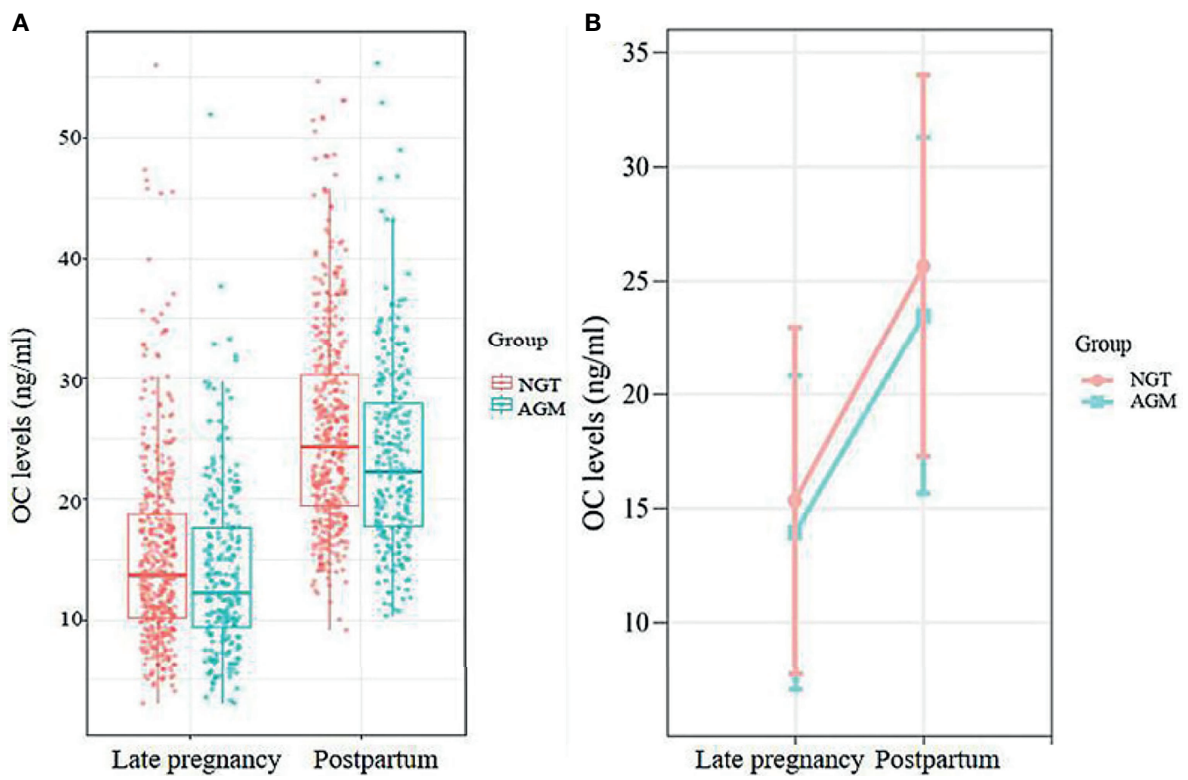

FIGURE 2 | (A) The distributions of OC levels at late pregnancy and postpartum in the NGT and AGM group. (B) Longitudinal change in OC levels in postpartum AGM individuals (orange line) and NGT individuals (blue line), adjusted for maternal age, parity, family history of DM, pre-BMl, and HbA1c in late pregnancy.

the other hand, infusion via subcutaneous minipump or daily injections or oral administration of recombinant OC significantly improved glucose tolerance and insulin sensitivity in mice fed a normal diet, which was possibly attributed to an increase in both $\beta$ cell mass and insulin secretion (27-29). In addition, OC stimulates pancreatic $\beta$-cell proliferation in cultured human islets (30). In accordance with previous animal studies, two meta-analyses confirmed that lower OC levels were observed in patients with type 2 diabetes than in normal controls $(7,31)$ and acknowledged that $\mathrm{OC}$ was negatively associated with fasting plasma glucose levels, $\mathrm{HbA1c}$, insulin resistance and body mass index (BMI) but positively correlated with improved glycemic control, weight loss and regular exercise $(11,12)$.

Pregnancy itself was an insulin-resistant physiological state, and by the end of pregnancy, insulin sensitivity decreased by roughly 50\% (32). To maintain euglycemia, insulin secretion increased 3 to 3.5 -fold to protect against insulin resistance (33). After delivery, women's insulin sensitivity increased rapidly by $120 \%$ compared with that during late pregnancy. However, women with previous GDM remained in a state of chronic inflammation and insulin sensitivity did not significantly improve (34). In the long run, individuals with a history of GDM seemed to have an approximately 10 times higher risk of developing T2DM than those with NGT during pregnancy (35). An increasing number of studies have explored the role of OC in GDM previously, but many of them focused on the difference in OC levels between GDM patients and normal controls $(21,23)$. There were limited studies on the relationship between OC changes and postpartum glucose metabolism in GDM patients.

Winhofer et al. (21) found that OC levels increased in all women at 12 weeks postpartum, which was confirmed in
Saucedo et al. research (36). However, fewer than 100 women with GDM underwent postpartum follow-up in their studies. In our study, we included more than 700 GDM patients. We found a 35.4\% incidence of AGM; among the individuals with AGM, $13.3 \%$ were diagnosed with diabetes at 6-8 weeks postpartum. Consistent with the previous research by Saucedo et al. (36), our study showed that in the GDM group, subjects who progressed to AGM at postpartum had lower OC concentrations than individuals in the NGT group. Furthermore, we found that the OC levels from late pregnancy to postpartum were consistently lower in AGM group than in NGT group. Considering that OC can stimulate insulin secretion and has been shown to have a beneficial effect on glucose metabolism in animal and human studies, we speculated that the consistently lower levels in OC in the postpartum AGM group was an insufficient compensation for insulin resistance.

To test this hypothesis, we conducted simple correlations and found that serum OC was positively related with HOMA- $\beta$ and the positive association was still robust after adjusting for age, postpartum BMI, parity, family history of diabetes and HbAlc in late pregnancy. Multivariate regression models further revealed that the risk of progressing to postpartum AGM decreased by $3.6 \%$ with per $1 \mathrm{ng} / \mathrm{ml}$ increment of serum OC in late pregnancy (OR:0.964, 95\%CI:0.940-0.988). Therefore, it is likely that in GDM patients, OC increases as a protective compensation mechanism to stimulate insulin secretion to cope with increased insulin demand and to further prevent developing of AGM.

There are several limitations in our study. First, we measured only the N-MID OC not ucOC, the bioactive form of OC, which is difficult to measure $(37,38)$. However, N-MID OC is the most 
stable form of OC in serum (26). Second, we observed the relationship between OC and postpartum AGM in only a short period. Therefore, it is necessary to conduct prospective and mechanistic studies in the future.

In conclusion, consistently low levels of osteocalcin from late pregnancy to postpartum in GDM patients were at high risk of postpartum AGM. Increasing serum OC levels may become a potential preventive indicator to curb the progression to postpartum IFG/IGT or even T2DM.

\section{DATA AVAILABILITY STATEMENT}

The raw data supporting the conclusions of this article will be made available by the authors, without undue reservation.

\section{ETHICS STATEMENT}

The studies involving human participants were reviewed and approved by The institutional ethics committee of Shanghai General Hospital. The ethics committee waived the requirement of written informed consent for participation.

\section{AUTHOR CONTRIBUTIONS}

YG and NL conceived of the design of the study and drafted the manuscript. ML, FF, and JY contributed to the data collection.

\section{REFERENCES}

1. Zoch ML, Clemens TL, Riddle RC. New Insights Into the Biology of Osteocalcin. Bone (2016) 82:42-9. doi: 10.1016/j.bone.2015.05.046

2. Al-Suhaimi EA, Al-Jafary MA. Endocrine Roles of Vitamin K-DependentOsteocalcin in the Relation Between Bone Metabolism and Metabolic Disorders. Rev Endocr Metab Disord (2020) 21:117-25. doi: 10.1007/ s11154-019-09517-9

3. Hauschka PV, Lian JB, Cole DE, Gundberg CM. Osteocalcin and Matrix Gla Protein: Vitamin K-Dependent Proteins in Bone. Physiol Rev (1989) 69:9901047. doi: 10.1152/physrev.1989.69.3.990

4. Morris DP, Stevens RD, Wright DJ, Stafford DW. Processive Post-Translational Modification. Vitamin K-Dependent Carboxylation of a Peptide Substrate. J Biol Chem (1995) 270:30491-8. doi: 10.1074/jbc.270.51.30491

5. Massera D, Biggs ML, Walker MD, Mukamal KJ, Ix JH, Djousse L, et al. Biochemical Markers of Bone Turnover and Risk of Incident Diabetes in Older Women: The Cardiovascular Health Study. Diabetes Care (2018) 41:1901-8. doi: 10.2337/dc18-0849

6. Guo H, Wang C, Jiang B, Ge S, Cai J, Zhou Y, et al. Association of Insulin Resistance and $\beta$-Cell Function With Bone Turnover Biomarkers in Dysglycemia Patients. Front Endocrinol (Lausanne) (2021) 12:554604. doi: 10.3389/fendo.2021.554604

7. Kunutsor SK, Apekey TA, Laukkanen JA. Association of Serum Total Osteocalcin With Type 2 Diabetes and Intermediate Metabolic Phenotypes: Systematic Review and Meta-Analysis of Observational Evidence. Eur J Epidemiol (2015) 30:599-614. doi: 10.1007/s10654-015-0058-x

8. Mizokami A, Mukai S, Gao J, Kawakubo-Yasukochi T, Otani T, Takeuchi H, et al. GLP-1 Signaling Is Required for Improvement of Glucose Tolerance by Osteocalcin. J Endocrinol (2020) 244:285-96. doi: 10.1530/joe-19-0288

9. Pi M, Kapoor K, Ye R, Nishimoto SK, Smith JC, Baudry J, et al. Evidence for Osteocalcin Binding and Activation of GPRC6A in $\beta$-Cells. Endocrinology (2016) 157:1866-80. doi: 10.1210/en.2015-2010

10. Lee NK, Sowa H, Hinoi E, Ferron M, Ahn JD, Confavreux C, et al. Endocrine Regulation of Energy Metabolism by the Skeleton. Cell (2007) 130:456-69. doi: 10.1016/j.cell.2007.05.047
MK and TS participated in the data analysis. YW and YP critically reviewed the data and the manuscript. All authors have reviewed and approved the final manuscript, contributed to the design of the study and interpretation of the data.

\section{FUNDING}

National Natural Science Foundation of China (No. 81870610 \& No. 82170879), the Shanghai Science and Technology Commission Foundation (No. 21Y11904800), Clinical Research Plan of SHDC (No. SHDC2020CR3065B).

\section{ACKNOWLEDGMENTS}

The authors acknowledge the contributions of all the participants.

\section{SUPPLEMENTARY MATERIAL}

The Supplementary Material for this article can be found online at: https://www.frontiersin.org/articles/10.3389/fendo.2022.803624/ full\#supplementary-material

11. Kord-Varkaneh H, Djafarian K, Khorshidi M, Shab-Bidar S. Association Between Serum Osteocalcin and Body Mass Index: A Systematic Review and Meta-Analysis. Endocrine (2017) 58:24-32. doi: 10.1007/s12020-0171384-4

12. Hiam D, Landen S, Jacques M, Voisin S, Alvarez-Romero J, Byrnes E, et al. Osteocalcin and Its Forms Respond Similarly to Exercise in Males and Females. Bone (2021) 144:115818. doi: 10.1016/j.bone.2020.115818

13. World Health Organization. Definition, Diagnosis and Classification of Diabetes Mellitus and Its Complications. Part 1: Diagnosis and Classification of Diabetes Mellitus. Geneva, Switzerland: WHO (1999).

14. American Diabetes Association. 2. Classification and Diagnosis of Diabetes: Standards of Medical Care in Diabetes-2018. Diabetes Care (2018) 41:S13-27. doi: $10.2337 / \mathrm{dc} 18-\mathrm{S} 002$

15. Lowe WLJr., Scholtens DM, Kuang A, Linder B, Lawrence JM, Lebenthal Y, et al. Hyperglycemia and Adverse Pregnancy Outcome Follow-Up Study (HAPO FUS): Maternal Gestational Diabetes Mellitus and Childhood Glucose Metabolism. Diabetes Care (2019) 42:372-80. doi: 10.2337/dc18-1646

16. Lowe WL Jr, Scholtens DM, Lowe LP, Kuang A, Nodzenski M, Talbot O, et al. Association of Gestational Diabetes With Maternal Disorders of Glucose Metabolism and Childhood Adiposity. Jama (2018) 320:1005-16. doi: 10.1001/jama.2018.11628

17. Yu Y, Arah OA, Liew Z, Cnattingius S, Olsen J, Sørensen HT, et al. Maternal Diabetes During Pregnancy and Early Onset of Cardiovascular Disease in Offspring: Population Based Cohort Study With 40 Years of Follow-Up. BMJ (2019) 367:16398. doi: 10.1136/bmj.16398

18. Aroda VR, Christophi CA, Edelstein SL, Zhang P, Herman WH, BarrettConnor E, et al. The Effect of Lifestyle Intervention and Metformin on Preventing or Delaying Diabetes Among Women With and Without Gestational Diabetes: The Diabetes Prevention Program Outcomes Study 10-Year Follow-Up. J Clin Endocrinol Metab (2015) 100:1646-53. doi: 10.1210/jc.2014-3761

19. Bao W, Li S, Chavarro JE, Tobias DK, Zhu Y, Hu FB, et al. Low CarbohydrateDiet Scores and Long-Term Risk of Type 2 Diabetes Among Women With a History of Gestational Diabetes Mellitus: A Prospective Cohort Study. Diabetes Care (2016) 39:43-9. doi: 10.2337/dc15-1642 
20. American Diabetes Association. 14. Management of Diabetes in Pregnancy: Standards of Medical Care in Diabetes-2021. Diabetes Care (2021) 44:S20010. doi: $10.2337 / \mathrm{dc} 21-\mathrm{S} 014$

21. Winhofer Y, Handisurya A, Tura A, Bittighofer C, Klein K, Schneider B, et al. Osteocalcin Is Related to Enhanced Insulin Secretion in Gestational Diabetes Mellitus. Diabetes Care (2010) 33:139-43. doi: 10.2337/dc09-1237

22. Srichomkwun P, Houngngam N, Pasatrat S, Tharavanij T, Wattanachanya L, Khovidhunkit W. Undercarboxylated Osteocalcin Is Associated With Insulin Resistance, But Not Adiponectin, During Pregnancy. Endocrine (2016) 53:129-35. doi: 10.1007/s12020-015-0829-X

23. Tabatabaei N, Giguère Y, Forest JC, Rodd CJ, Kremer R, Weiler HA. Osteocalcin Is Higher Across Pregnancy in Caucasian Women With Gestational Diabetes Mellitus. Can J Diabetes (2014) 38:307-13. doi: 10.1016/j.jcjd.2014.02.007

24. Metzger BE, Gabbe SG, Persson B, Buchanan TA, Catalano PA, Damm P, et al. International Association of Diabetes and Pregnancy Study Groups Recommendations on the Diagnosis and Classification of Hyperglycemia in Pregnancy. Diabetes Care (2010) 33:676-82. doi: 10.2337/dc09-1848

25. Alberti KG, Zimmet PZ. Definition, Diagnosis and Classification of Diabetes Mellitus and Its Complications. Part 1: Diagnosis and Classification of Diabetes Mellitus Provisional Report of a Who Consultation. Diabetes Med (1998) 15:539-53. doi: 10.1002/(sici)1096-9136(199807)15:7<539::Aiddia668>3.0.Co;2-s

26. Nagasue K, Inaba M, Okuno S, Kitatani K, Imanishi Y, Ishimura E, et al. Serum $\mathrm{N}$-Terminal Midfragment vs. Intact Osteocalcin Immunoradiometric Assay as Markers for Bone Turnover and Bone Loss in Hemodialysis Patients. BioMed Pharmacother (2003) 57:98-104. doi: 10.1016/s0753-3322(02)00344-x

27. Ferron M, McKee MD, Levine RL, Ducy P, Karsenty G. Intermittent Injections of Osteocalcin Improve Glucose Metabolism and Prevent Type 2 Diabetes in Mice. Bone (2012) 50:568-75. doi: 10.1016/j.bone.2011.04.017

28. Zhou B, Li H, Liu J, Xu L, Guo Q, Zang W, et al. Autophagic Dysfunction Is Improved by Intermittent Administration of Osteocalcin in Obese Mice. Int J Obes (Lond) (2016) 40:833-43. doi: 10.1038/ijo.2016.1

29. Yasutake Y, Mizokami A, Kawakubo-Yasukochi T, Chishaki S, Takahashi I, Takeuchi $\mathrm{H}$, et al. Long-Term Oral Administration of Osteocalcin Induces Insulin Resistance in Male Mice Fed a High-Fat, High-Sucrose Diet. Am J Physiol Endocrinol Metab (2016) 310:E662-75. doi: 10.1152/ajpendo.00334.2015

30. Sabek OM, Nishimoto SK, Fraga D, Tejpal N, Ricordi C, Gaber AO. Osteocalcin Effect on Human $\beta$-Cells Mass and Function. Endocrinology (2015) 156:3137-46. doi: 10.1210/en.2015-1143

31. Liu C, Wo J, Zhao Q, Wang Y, Wang B, Zhao W. Association Between Serum Total Osteocalcin Level and Type 2 Diabetes Mellitus: A Systematic Review and Meta-Analysis. Horm Metab Res (2015) 47:813-9. doi: 10.1055/s-00351564134
32. Catalano PM, Tyzbir ED, Roman NM, Amini SB, Sims EA. Longitudinal Changes in Insulin Release and Insulin Resistance in Nonobese Pregnant Women. Am J Obstet Gynecol (1991) 165:1667-72. doi: 10.1016/0002-9378 (91)90012-g

33. Agha-Jaffar R, Oliver N, Johnston D, Robinson S. Gestational Diabetes Mellitus: Does an Effective Prevention Strategy Exist? Nat Rev Endocrinol (2016) 12:533-46. doi: 10.1038/nrendo.2016.88

34. McIntyre HD, Catalano P, Zhang C, Desoye G, Mathiesen ER, Damm P. Gestational Diabetes Mellitus. Nat Rev Dis Primers (2019) 5:47. doi: 10.1038/ s41572-019-0098-8

35. Vounzoulaki E, Khunti K, Abner SC, Tan BK, Davies MJ, Gillies CL. Progression to Type 2 Diabetes in Women With a Known History of Gestational Diabetes: Systematic Review and Meta-Analysis. BMJ (2020) 369:m1361. doi: 10.1136/bmj.m1361

36. Saucedo R, Rico G, Vega G, Basurto L, Cordova L, Galvan R, et al. Osteocalcin, Under-Carboxylated Osteocalcin and Osteopontin Are Not Associated With Gestational Diabetes Mellitus But Are Inversely Associated With Leptin in Non-Diabetic Women. J Endocrinol Invest (2015) 38:519-26. doi: 10.1007/ s40618-014-0220-4

37. Liu JM, Rosen CJ, Ducy P, Kousteni S, Karsenty G. Regulation of Glucose Handling by the Skeleton: Insights From Mouse and Human Studies. Diabetes (2016) 65:3225-32. doi: 10.2337/db16-0053

38. Delmas PD, Eastell R, Garnero P, Seibel MJ, Stepan J. The Use of Biochemical Markers of Bone Turnover in Osteoporosis. Committee of Scientific Advisors of the International Osteoporosis Foundation. Osteoporos Int (2000) 11(Suppl 6):S2-17. doi: 10.1007/s001980070002

Conflict of Interest: The authors declare that the research was conducted in the absence of any commercial or financial relationships that could be construed as a potential conflict of interest.

Publisher's Note: All claims expressed in this article are solely those of the authors and do not necessarily represent those of their affiliated organizations, or those of the publisher, the editors and the reviewers. Any product that may be evaluated in this article, or claim that may be made by its manufacturer, is not guaranteed or endorsed by the publisher.

Copyright (C) 2022 Gong, Li, Lai, Fang, Yang, Kang, Shen, Peng and Wang. This is an open-access article distributed under the terms of the Creative Commons Attribution License (CC BY). The use, distribution or reproduction in other forums is permitted, provided the original author(s) and the copyright owner(s) are credited and that the original publication in this journal is cited, in accordance with accepted academic practice. No use, distribution or reproduction is permitted which does not comply with these terms. 